\title{
Ability of the ALBI grade to predict posthepatectomy liver failure and long- term survival after liver resection for different BCLC stages of HCC
}

\author{
Ze-Qun Zhang, Li Xiong, Jiang-Jiao Zhou, Xiong-Ying Miao, Qing-Long Li, Yu Wen ${ }^{*}$ and Heng Zou* (1)
}

\begin{abstract}
Background: Underlying liver function is a major concern when applying surgical resection for hepatocellular carcinoma (HCC). We aimed to explore the capability of the albumin-bilirubin (ALBI) grade to predict post-hepatectomy liver failure (PHLF) and long-term survival after hepatectomy for HCC patients with different Barcelona Clinic Liver Cancer (BCLC) stages.
\end{abstract}

Methods: Between January 2010 and December 2014, 338 HCC patients who were treated with liver resection were enrolled. The predictive accuracy of ALBI grade system for PHLF and long-term survival across different BCLC stages was examined.

Results: A total of 26 (7.7\%) patients developed PHLF. Patients were divided into BCLC O/A and BCLC B/C categories. $A L B I$ score was found to be a strong independent predictor of PHLF across different BCLC stages by multivariate analysis. In terms of overall survival (OS), it exhibited high discriminative power in the total cohort and in BCLC 0/ A subgroup. However, differences in OS between ALBI grade 1 and 2 patients in BCLC B/C subgroup were not significant $(P=0.222)$.

Conclusion: The ALBI grade showed good predictive ability for PHLF in HCC patients across different BCLC stages. However, the ALBI grade was only a significant predictor of OS in BCLC stage 0/A patients and failed to predict OS in BCLC stage B/C patients.

Keywords: Child-Pugh grade, Post-hepatectomy liver failure, Albumin-bilirubin score, Hepatocellular carcinoma, BCLC classification, Overall survival

\section{Background}

As one of the most common and aggressive malignancies, hepatocellular carcinoma (HCC) ranks the second most fatal cancer globally [1]. Although surgical resection is the mainstay therapy for extremely early and early stage $\mathrm{HCC}$, it is not recommended for intermediate and advanced stage $\mathrm{HCC}$ as determined by Barcelona Clinic Liver Cancer (BCLC) classification because of the increased risks and limited advantages of the procedure

\footnotetext{
* Correspondence: wenyu2861@csu.edu.cn; zhcsuxy@csu.edu.cn Ze-Qun Zhang and Li Xiong are co-first authors.

Ze-Qun Zhang and Li Xiong contributed equally to this work.

Department of General Surgery, The Second Xiangya Hospital, Central South University, Changsha 410011, Hunan, China
}

[2]. Recently, due to advances in techniques and perioperative management, surgical resection for HCC has become more aggressive. $\mathrm{HCC}$ patients at BCLC B and $\mathrm{C}$ stages have been reported to have good prognosis after surgical resection [3-6].

Patients with BCLC B and C HCC often possess a high tumor burden, including an increased tumor size, multiple tumors, and vascular invasion. To achieve microscopically radical resection margins, patients tend to undergo extended liver resection, which increases the chances of post-hepatectomy liver failure (PHLF). This is especially obvious when patients have underlying longterm hepatic disorders, such as hepatic fibrosis and cirrhosis [7-9], which often result in impaired liver

(c) The Author(s). 2018 Open Access This article is distributed under the terms of the Creative Commons Attribution 4.0 International License (http://creativecommons.org/licenses/by/4.0/), which permits unrestricted use, distribution, and 
function. As a life-threatening complication with an intrinsic risk of mortality, PHLF is still a major concern for hepatic surgeons in clinical practice. To minimize PHLF and postoperative mortality, development of a simple, objective, and accurate assessment tool for liver function prior to surgery is of vital importance. Recently, the application of albumin-bilirubin (ALBI) grade to evaluate liver function in patients with HCC was proposed [10-12]. The ALBI grade not only offers similar prognostic information as the Child-Pugh $(\mathrm{CP})$ class but also eliminates the necessity of assessing empirical variables such as hepatic encephalopathy and ascites. Several studies have shown that the ALBI grade had a superior predictive value for PHLF and overall survival (OS) in HCC patients following liver resection [13, 14].

The survival of HCC patients undergoing liver resection depends mainly on tumor burden and hepatic function [15]. The ALBI grade was proven to be a reliable hepatic function assessment tool and could accurately predict survival in patients with extremely early or early stage HCC who underwent liver resection [12, 16-19]. However, few studies have examined the role of the ALBI grade in predicting the survival of patients with intermediate and advanced stage $\mathrm{HCC}$ after hepatectomy.

Here, we evaluated the effectiveness of the ALBI grade in predicting PHLF and OS across different BCLC stages among HCC patients undergoing liver resection.

\section{Methods}

\section{Patients}

In this retrospective study, HCC patients who underwent liver resection were enrolled from January 2010 to December 2014 at the Second Xiangya Hospital. The hospital medical database was searched to retrieve baseline parameters. Inclusion criteria included the following: liver function with CP A or B; no other simultaneous malignancies; no therapy for HCC before surgery; and no insufficiency concerning the heart, lung, kidney, and brain before operation. Written informed consent for this retrospective research was waived. This investigation was approved by the ethics committee of the hospital.

\section{Diagnosis and definitions}

HCC was diagnosed using histopathological examination of the surgical samples. The HCC stage accorded with the BCLC guidelines [20]. Based on the recommendations of the International Study Group of Liver Surgery (ISGLS), PHLF was defined as a total serum bilirubin value $>50 \mu \mathrm{mol} \mathrm{l}^{-1}$ on day 5 after surgery or hereafter and a prothrombin time index $<50 \%$ (an international normalized ratio (INR) > 1.7) in the meanwhile [21, 22]. Adjustment in clinical administration was not required for grade A PHLF; non-invasive interventions such as fresh-frozen plasma, albumin management, diuretics, and ventilation were applied for grade B PHLF; and invasive interventions such as circulatory and extracorporeal liver support, hemodialysis, intubation, and mechanical ventilation were used barely for grade C PHLF [21]. A relative low platelet count $\left(<100 \times 10^{9} / \mathrm{l}\right)$ with splenomegaly and diagnosis of gastric/esophageal varices were used as indicators of clinically significant portal hypertension (CSPH) $[23,24]$. Hepatectomy was defined as minor if fewer than three Couinaud segments were resected and major if three liver segments or more were resected. Deaths recorded within 60 days after surgery were considered to represent mortality.

Determination of the CP class was performed according to methods published previously [25]. The model of end-stage liver disease (MELD) score was computed applying the formula: $9.57 \times \ln ($ creatinine $[\mathrm{mg} / \mathrm{dl}])+$ $11.2 \times \ln (\mathrm{INR})+3.78 \times \ln ($ bilirubin $[\mathrm{mg} / \mathrm{dl}])+6.43$ [26] The calculation of the ALBI score used this equation: ALBI score $=-0.085 \times\left(\right.$ albumin $\left.\left[g^{-1}\right]\right)+0.66 \times \log _{10}(-$ total bilirubin $\left[\mu \mathrm{mol} \mathrm{l} \mathrm{l}^{-1}\right]$ ), and was further categorized into three different grades: grade $3(>-1.39)$, grade 2 $(>-2.60$ to $\leq-1.39)$, and grade $1(\leq-2.60)[10]$.

\section{Surgical technique}

Before surgery, contrast-enhanced computed tomography $(\mathrm{CT})$ and abdominal ultrasound were routinely conducted to assess tumor condition. Preoperative indocyanine green tests were routinely performed for patients with hepatitis B or C infection. For laparotomy, right subcostal margin incision was chosen. Laparoscopic approach was implemented widely for tumor diameter $<5 \mathrm{~cm}$ located in segments $2-6$. During operation, the fluid infusion was minimal to maintain a relatively low central venous pressure to reduce bleeding. To precisely determine the relationship between tumors and other tissues as well as assess the orientation of tumors, intraoperative ultrasound was conducted if needed. If resectability had been determined, anatomical resection was performed aiming to excise the tumor's portal territory when future remnant liver functional reserve was sufficient.

\section{Follow-up evaluation}

The trace of each patient was done at 1 month following discharge from hospital and at intervals of 3 months during the first year, and at 6-month intervals in following years. Follow-up included liver function tests, determination of $\alpha$-fetoprotein (AFP) concentration, and abdominal-enhanced CT. The primary endpoints for this study were PHLF and death. OS was from date of resection to last living visit or loss to follow-up. The last visit was done on December 31, 2017. 


\section{Statistical analysis}

Continuous data were analyzed by $t$ test or Mann-Whitney $U$ test. $X^{2}$ test was used to compare categorical data. Identification of independent predictors of PHLF was achieved by multivariate logistic regression analysis. Receiver operating characteristic (ROC) curve analysis was carried out to determine the cut-off points for the occurrence of PHLF. The area under the ROC curve (AUC) was applied to assess discriminative power. Comparisons between ROC curves were conducted with Delong test. OS was assessed visually through Kaplan-Meier plots, and differences between curves were analyzed by log-rank test. Independent risk factors for OS were identified using multivariate Cox proportional hazard regression models. Statistical significance was considered for a two-tailed value of $P<0.05$. SPSS 17.0 (Inc., Chicago, IL, USA) was applied for data analysis.

\section{Results}

\section{Patient information}

A sample of $338 \mathrm{HCC}$ patients was included. Most patients had a CP class of A $(308 / 338,91.1 \%)$, and the remaining 30 were class B $(30 / 308,8.9 \%)$. For ALBI grade 1 , there were $39.6 \%(134 / 338)$ patients while grade 2 had 58.6\% patients (198/338) and grade 3 had 1.8\% (6/ 338) patients. Table 1 shows the features of the patients.

\section{Postoperative morbidity, PHLF, and mortality}

Of the 338 patients, 142 (42.0\%) developed complications after surgery. Pneumonia which had the highest frequency occurred in 39 patients (11.5\%), followed by plural effusion and ascites in 37 (10.9\%). PHLF occurred in 26 patients $(7.7 \%)$, among them, 8 patients $(2.4 \%)$ had grade A PHLF, 13 patients (3.8\%) had grade B, and 5 patients $(1.5 \%)$ had grade C. During 60 days after operation, 15 patients died, resulting in a postoperative mortality rate of $4.4 \%$.

\section{Correlation between PHLF and CP class or ALBI grade}

PHLF occurred in 16 of the 308 (5.2\%) CP class A patients and in 10 of the $30(33.3 \%) \mathrm{CP}$ class B patients $(P<0.001)$. Among the total cohort, 3 of $134(2.2 \%)$ ALBI grade 1 patients had PHLF, while 19 of 198 (9.6\%) ALBI grade 2 patients fell into PHLF $(P=0.008)$. Four of 6 (66.7\%) ALBI grade 3 patients developed PHLF. Higher ALBI grade led to higher risk of grade C PHLF (Fig. 1a). The incidence of grade C PHLF was higher in patients with ALBI grade 3 compared to those with ALBI grade $2(P=0.003)$ or ALBI grade $1(P=0.001)$. Moreover, patients with BCLC B/C HCC were more likely to suffer from grade $C$ PHLF than those with BCLC 0/A HCC ( $P=0.027$, Fig. 1b).
Multivariate analyses of PHLF across the BCLC stages of HCC The entire cohort was categorized into two subgroups in the multivariate logistic regression analysis. For the total cohort, ALBI score, CP score, and major hepatectomy were identified significant. In the BCLC 0/A subgroup, ALBI score, CP score, and major hepatectomy were identified significant. In the BCLC B/C subgroup, platelet count, ALBI score, and CP score were identified significant (Table 2).

\section{Discriminative power of ALBI score to predict PHLF across the BCLC stages of HCC}

Figure 2a shows the predictive power of -ALBI scores for PHLF as determined by the ROC curve analyses which were the total cohort (AUC, 0.782; 95\% CI, 0.701-0.862, $P<0.001$ ), BCLC $0 / \mathrm{A}$ subgroup (AUC, 0.780; 95\% CI, 0.670-0.889; $P<0.001)$, and BCLC B/C subgroup (AUC, 0.790; 95\% CI, 0.680-0.900; $P=0.002$ ). For the total cohort, the -ALBI score had a greater AUC than CP score (AUC, 0.656; 95\% CI, 0.527-0.784; $P=0.008) \quad(P=0.005$, Delong test $)$ and MELD score (AUC, 0.669; 95\% CI, 0.566-0.771; $P=0.004)(P=0.013$, Delong test) (Fig. 2b). The -ALBI score had an optimal cut-off value of 2.44 (that was to say, the cut-off point of ALBI score lay in -2.44 , the same below), presenting a specificity and a sensitivity of $56.1 \%$ and $88.5 \%$ respectively.

We divided the entire cohort into two subgroups, BCLC $0 / A$ and BCLC $\mathrm{B} / \mathrm{C}$, to minimize the potential confounding bias caused by tumor characteristics. The cut-off value of the former group was 2.41, with a specificity and a sensitivity of $60.3 \%$ and $81.3 \%$ respectively, while the cut-off value of the latter group was 2.36 , with a sensitivity of $90.0 \%$ and a specificity of $60.2 \%$. In the BCLC 0/A group, the AUC of -ALBI score was greater than MELD and CP scores (Fig. 2c). Same result could be obtained in BCLC B/C subgroup (Fig. 2d).

\section{Discriminative power of the ALBI grade for OS at different BCLC stages of HCC}

A total of 183 HCC deaths (54.1\%) occurred throughout the follow-up (with a median of 31.5 months). The OS rate in the total cohort at 1,2 , and 3 years were $78.1 \%$, $55.3 \%$, and $49.4 \%$, in that order. The OS rate in patients with ALBI grade 1 at 1,2 , and 3 years were $83.6 \%, 68.7 \%$, and $61.2 \%$, respectively, which were higher compared to patients with ALBI grade 2 (74.7\%, 47.0\%, and 41.9\%, respectively) $(P=0.003)$ (Fig. 3a). We did not analyze the OS of the ALBI grade 3 cohort because there were few patients (6 patients) in this group. Four of these patients died within 2 years after the operation. In the BCLC 0/A subgroup, the OS rate of ALBI grade 2 patients was lower compared to that of ALBI grade 1 patients $(P=0.008)$ 
Table 1 Baseline characteristics of 338 HCC patients

\begin{tabular}{|c|c|c|c|}
\hline Variables & $\begin{array}{l}\text { Total cohort } \\
(n=338)\end{array}$ & $\begin{array}{l}\text { BCLC 0/A HCC } \\
(n=205)\end{array}$ & $\begin{array}{l}\mathrm{BCLC} \mathrm{B/C} \mathrm{HCC} \\
(n=133)\end{array}$ \\
\hline $\mathrm{Age}$ years ${ }^{\dagger}$ & $52(44-66)$ & $52(44-60)$ & $52(43-60)$ \\
\hline Male gender ${ }^{\ddagger}$ & $299(88.5)$ & $180(87.5)$ & $119(89.5)$ \\
\hline Positive $\mathrm{HBsAg}^{\ddagger}$ & $278(82.2)$ & $170(82.9)$ & $108(81.2)$ \\
\hline Total bilirubin, $\mu \mathrm{mol} / /^{\dagger}$ & $14.0(13.4-19.4)$ & $13.9(10.0-19.6)$ & $14.4(10.6-18.7)$ \\
\hline Albumin, $g / l^{+}$ & $37.9(35.1-40.8)$ & $38.0(35.4-41.0)$ & $37.6(34.0-40.7)$ \\
\hline$A L T, U / I^{+}$ & $36.1(25.8-52.0)$ & $34.3(24.7-53.0)$ & $37.9(26.4-51.0)$ \\
\hline Prothrombin time, $\mathrm{s}^{\dagger}$ & $13.2(12.0-14.1)$ & $13.2(12.0-14.1)$ & $13.2(12.1-14.1)$ \\
\hline $\mathrm{INR}^{\dagger}$ & $1.04(0.95-1.14)$ & $1.04(0.94-1.14)$ & $1.05(0.95-1.14)$ \\
\hline Platelet count, $\times 10^{9} /\left.\right|^{\dagger}$ & $155(110-205)$ & $155(110-211)$ & $154(113.5-201.5)$ \\
\hline Maximum tumor size, $\mathrm{cm}^{+}$ & $6.0(4.2-10.0)$ & $6.0(3.5-9.2)$ & $8(5.0-11.0)$ \\
\hline \multicolumn{4}{|l|}{ Serum AFP, ng/ml ${ }^{\ddagger}$} \\
\hline$\geq 400$ & $141(41.7)$ & 73 (35.6) & $68(51.1)$ \\
\hline$<400$ & $197(58.3)$ & $132(64.4)$ & 65 (48.9) \\
\hline $\mathrm{CSPH}^{\ddagger}$ & $56(16.6)$ & $34(16.6)$ & $22(16.5)$ \\
\hline ALBI score ${ }^{\dagger}$ & $-2.460(-2.704--2.192)$ & $-2.518(-2.719--2.236)$ & $-2.399(-2.702--2.136)$ \\
\hline \multicolumn{4}{|l|}{ ALBI grade ${ }^{\ddagger}$} \\
\hline 1 & $134(39.6)$ & $89(43.4)$ & 45 (33.8) \\
\hline 2 & $198(58.6)$ & $114(55.6)$ & $84(63.2)$ \\
\hline 3 & $6(1.8)$ & $2(1.0)$ & $4(3.0)$ \\
\hline MELD score (range) & $7(6-18)$ & $7(6-18)$ & $7(6-17)$ \\
\hline \multicolumn{4}{|l|}{ MELD score $e^{\ddagger}$} \\
\hline$\geq 9$ & $88(26.0)$ & $47(22.9)$ & $41(30.8)$ \\
\hline$<9$ & $250(74.0)$ & $158(77.1)$ & $92(69.2)$ \\
\hline \multicolumn{4}{|l|}{ Child-Pugh grade ${ }^{\ddagger}$} \\
\hline A & $308(91.1)$ & $190(92.7)$ & $118(88.7)$ \\
\hline B & $30(8.9)$ & $15(7.3)$ & $15(11.3)$ \\
\hline C & $0(0)$ & $0(0)$ & $0(0)$ \\
\hline \multicolumn{4}{|l|}{$\mathrm{BCLC}_{\text {stage }}{ }^{\ddagger}$} \\
\hline 0 & $12(3.6)$ & - & - \\
\hline A & $193(57.1)$ & - & - \\
\hline B & $82(24.3)$ & - & - \\
\hline C & $51(15.1)$ & - & - \\
\hline
\end{tabular}

HCC hepatocellular carcinoma, $H B s A g$ hepatitis B surface antigen, $A L T$ alanine aminotransferase, INR international normalized ratio, $A F P$ a-fetoprotein, CSPH clinically significant portal pressure, $A L B I$ albumin-bilirubin, $B C L C$ Barcelona Clinic Liver Cancer †Values are median (interquartile range)

$\ddagger$ Values are number (\%)

(Fig. 3c). However, in BCLC B/C subgroup, OS did not differ between ALBI grade 2 patients and ALBI grade 1 patients $(P=0.222)$ (Fig. 3e). The OS exhibited little difference between patients with $\mathrm{CP}$ class $\mathrm{A}$ and those with $\mathrm{CP}$ class $\mathrm{B}$ in the total cohort $(P=0.052)$ (Fig. 3b), BCLC 0/A subgroup $(P=0.052)$ (Fig. $3 \mathrm{~d}$ ) and BCLC $\mathrm{B} / \mathrm{C}$ subgroup $(P=0.911)$ (Fig. 3f). Moreover, comparison of OS between MELD scores $<9$ and $\geq 9$ also showed no statistical difference $(P=0.784)$ (Additional file 1: Figure S1).

\section{Multivariate cox regression analyses of OS across BCLC stages}

Multivariate analyses revealed that the ALBI score, platelet count, tumor size, microvascular invasion (MVI), and differentiation grade were significant risk factors for OS in the total cohort. We further performed multivariate analysis in the two subgroups (Table 3). In the BCLC 0/ A subgroup, we observed similar results as in the total cohort (Table 3). Interestingly, the ALBI score was not a 

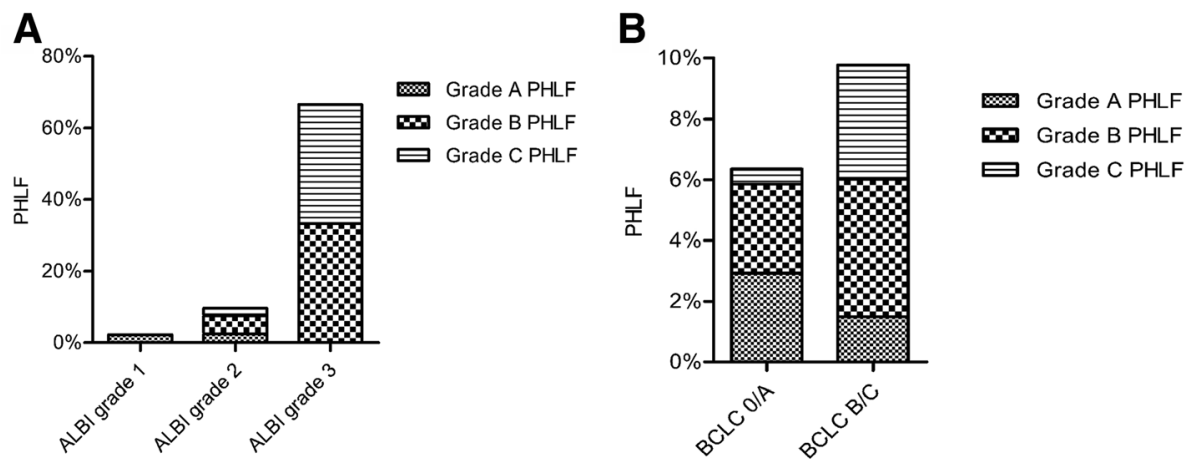

Fig. 1 Correlation between incidence and severity of PHLF and ALBI grade (a), and BCLC classification subgroups (b). PHLF, posthepatectomy liver failure; BCLC, Barcelona Clinic Liver Cancer

significant predictor, only MVI and differentiation grade remained independent predictors of OS in the BCLC B/ C subgroup (Table 3 ).

\section{Discussion}

Surgical resection is extensively performed for HCC patients with a favorable liver functional reserve. However, PHLF remains a serious complication of hepatic resection and a main cause of postoperative mortality [7, 27]. To improve the survival of patients with PHLF, early diagnosis and therapy are imperative. Many previous reports have provided evidence that the ALBI grade is an effective predictor of PHLF after liver resection in patients with HCC $[13,14]$. However, few studies have focused on the role of the ALBI score in predicting PHLF for patients with intermediate and advanced HCC. We found that the ALBI score could predict PHLF not only in the total cohort but also in the BCLC 0/A and BCLC $\mathrm{B} / \mathrm{C}$ subgroups. BCLC $\mathrm{B} / \mathrm{C}$ patients have a relatively higher tumor burden and often possess a larger tumor size, satellite nodes, or portal/hepatic vein tumor thrombosis. To achieve a radical cure, patients are inclined to undergo extensive or major hepatectomy in clinical practice, which increases the probability of occurrence of PHLF, especially for patients suffering from chronic hepatic disorders. For these reasons, accurate assessment of hepatic function prior to surgery is of great clinical significance for these patients.

To determine whether ALBI score can predict PHLF accurately, we used a stage-stratified approach. Patients with BCLC B/C HCC owned a greater possibility of occurring severer PHLF than those with BCLC 0/A HCC. Several facts may contribute to this result. On the one hand, generally, BCLC B/C HCC own a heavier tumor burden, it is easy to make extensive hepatectomy to achieve radical resection. On the other hand, BCLC B/C HCC have a longer time of tumor progression, so the liver function of these patients might be worse than that of BCLC 0/A patients. ROC curve analyses demonstrated that the AUC values of MELD and CP scores were lower compared to that of the -ALBI score in predicting PHLF in the total cohort and the BCLC stage subgroups, implying that the ALBI score might have better prognostic value for PHLF among

Table 2 Multivariate logistic regression analyses for posthepatectomy liver failure across BCLC stages

\begin{tabular}{|c|c|c|c|c|c|c|}
\hline \multirow[t]{3}{*}{ Variable } & \multicolumn{6}{|c|}{ Multivariate logistic regression } \\
\hline & \multicolumn{2}{|l|}{ Total cohort } & \multicolumn{2}{|l|}{$\mathrm{BCLC} 0 / \mathrm{A}$} & \multicolumn{2}{|l|}{$\mathrm{BCLC} B / \mathrm{C}$} \\
\hline & OR $(95 \% \mathrm{Cl})$ & $P$ & OR $(95 \% \mathrm{Cl})$ & $P$ & OR $(95 \% \mathrm{Cl})$ & $P$ \\
\hline $\begin{array}{l}\text { Prothrombin time } \\
>14 \mathrm{~s}\end{array}$ & $1.19(0.39-3.63)$ & 0.765 & $1.39(0.35-5.47)$ & 0.635 & $0.48(0.04-6.60)$ & 0.584 \\
\hline $\begin{array}{l}\text { Platelet count } \\
<100 \times 10^{9} / 1\end{array}$ & $2.82(0.97-8.19)$ & 0.056 & $0.45(0.07-2.94)$ & 0.405 & $132.70(6.04-916.91)$ & 0.002 \\
\hline Tumor size $>5 \mathrm{~cm}$ & $0.65(0.21-2.01)$ & 0.456 & $0.50(0.11-2.23)$ & 0.361 & $0.49(0.04-6.52)$ & 0.587 \\
\hline ALBI score $>-2.44$ & $3.43(1.11-10.57)$ & 0.032 & $2.33(1.09-9.19)$ & 0.046 & $30.48(1.36-682.73)$ & 0.031 \\
\hline Child-Pugh score $>6$ & $6.47(2.10-19.94)$ & 0.001 & $5.50(1.23-24.50)$ & 0.025 & $43.21(2.43-767.29)$ & 0.010 \\
\hline Blood loss $>400 \mathrm{ml}$ & $2.13(0.83-5.44)$ & 0.115 & $1.75(0.55-5.57)$ & 0.346 & $5.86(0.53-64.72)$ & 0.149 \\
\hline Major hepatectomy & $5.51(1.86-16.29)$ & 0.002 & $7.23(1.73-30.57)$ & 0.007 & $12.02(0.97-149.04)$ & 0.053 \\
\hline MELD score $>8$ & $0.67(0.21-2.11)$ & 0.496 & $0.90(0.21-3.78)$ & 0.885 & $0.35(0.03-3.62)$ & 0.378 \\
\hline
\end{tabular}


A

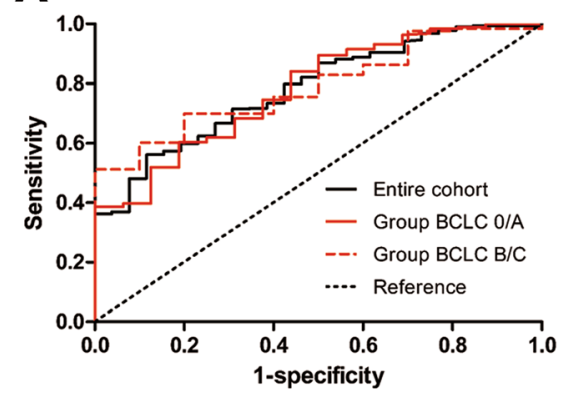

C

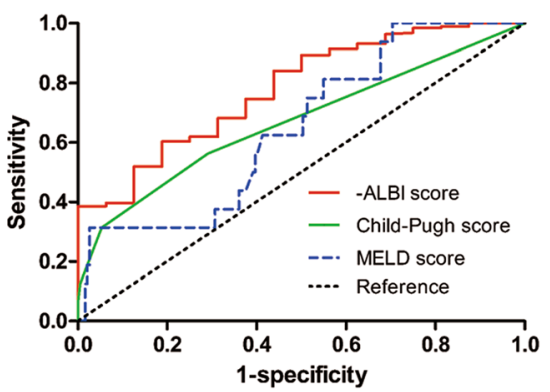

B

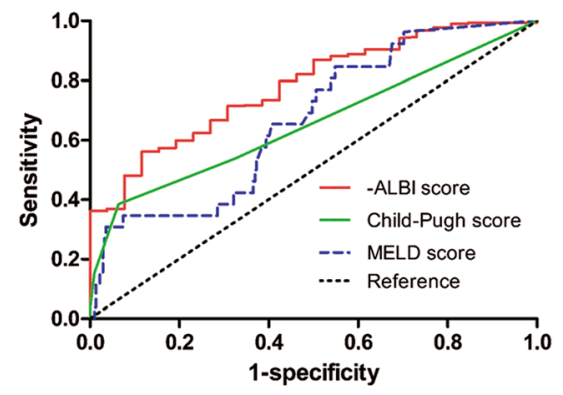

D

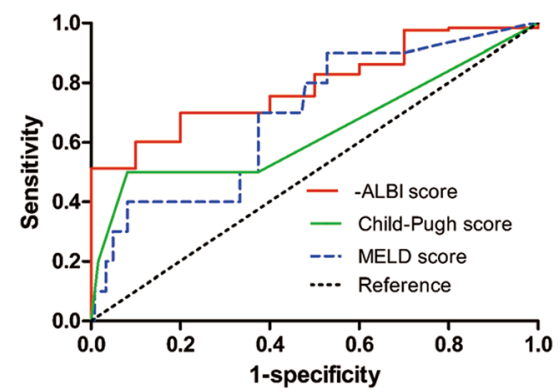

Fig. 2 Receiver operating characteristic (ROC) curve analyses of -ALBI scores for predicting PHLF in the entire cohort and the BCLC stage subgroups (a). ROC curves for Child-Pugh score, MELD score, and -ALBI score for predicting PHLF in the entire cohort (b), BCLC O/A subgroup (c), and BCLC B/C subgroup (d). ALBI, albumin-bilirubin; BCLC, Barcelona Clinic Liver Cancer; PHLF, posthepatectomy liver failure. MELD, model for end-stage liver disease

patients undergoing liver resection. This was consistent with previous reports $[13,14]$.

In addition, our results revealed that the $-\mathrm{ALBI}$ grade had an AUC of 0.790 for predicting PHLF in BCLC B/C patients, which indicates a relatively good prognostic value. The ROC curve showed that optimal cut-off value of -ALBI calculated by the ROC curve was 2.36 in BCLC $\mathrm{B} / \mathrm{C}$ HCC subgroup. This suggested that for patients with an ALBI score $>-2.36$ in BCLC B/C stage, increased attention should be given by hepatic surgeons to avoid PHLF. Additionally, the ALBI score showed a favorable prognostic value for PHLF in the total cohort and in the BCLC 0/A subgroup, which is consistent with the earlier studies $[16,17]$. This study indicated that the ALBI score might be helpful in choosing eligible candidates who may benefit from surgery, especially among BCLC B/C patients with a small-sized liver after resection. Moreover, we found MELD score showed no significance in the multivariate analysis for PHLF in this research. On the one hand, this might be on account of the small sample size from a single center. On the other hand, MELD score maybe suffers some limitations itself. For example, several other studies reported that MELD score showed no predictive power for perioperative outcomes in HCC patients without cirrhosis [28-30]. And interestingly, we found that the platelet count was an effective predictor of PHLF only in BCLC B/C patients, but not in BCLC 0/A patients. The platelet count is commonly thought to be correlated with the severity of portal hypertension [31]. Therefore, portal hypertension might play a more important role in predicting PHLF for $\mathrm{BCLC} \mathrm{B/C}$ patients than in those with early stage $\mathrm{HCC}$, but further study is required to verify this conclusion.

The survival of HCC patients was partly impacted by the underlying liver function. Consistent with the previous studies [16-18], we found that the ALBI score was a significant risk factor for OS in both the total cohort and the BCLC 0/A subgroup. Moreover, the OS rate was higher in ALBI grade 1 patients compared to that in ALBI grade 2 patients both in the total cohort and in the BCLC 0/A subgroup. However, OS showed little difference among $\mathrm{CP}$ classes in the total cohort and the two subgroups. These results suggested that the ALBI grade might have a greater discriminative ability than the CP class for predicting the OS of HCC patients after liver resection in a curative setting.

Furthermore, the prognostic value of ALBI scores was evaluated for BCLC B/C patients. According to the BCLC staging system in most Western countries, hepatectomy is not recommended for this unique group of patients [20]. However, recent studies have demonstrated that intermediate and advanced HCCs were not 

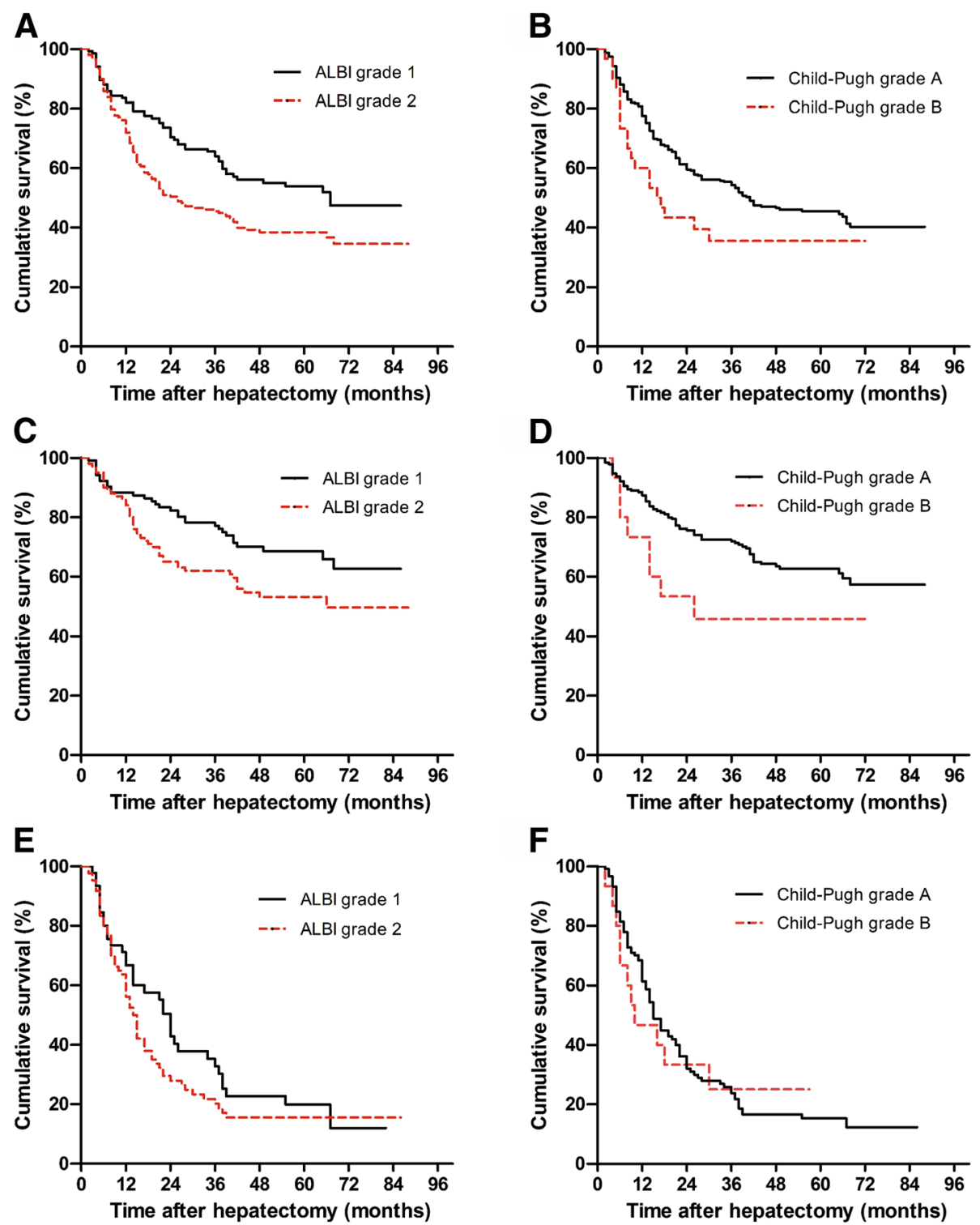

Fig. 3 Kaplan-Meier curves demonstrating overall survival according to the $\mathbf{a}$ ALBI grade in the total cohort, $\mathbf{b}$ Child-Pugh class in the total cohort, $\mathbf{c}$ ALBI grade in the BCLC O/A subgroup, $\mathbf{d}$ Child-Pugh class in the BCLC O/A subgroup, e ALBI grade in the BCLC B/C subgroup, and $\mathbf{f} C$ Child-Pugh class in the BCLC B/C subgroup. ALBI, albumin-bilirubin; BCLC, Barcelona Clinic Liver Cancer

absolute contradictions for surgical resection, which could provide significant survival benefits for carefully selected patients [6]. Importantly, we found that OS showed little difference between ALBI grade 2 and ALBI grade 1 patients in this cohort $(P=0.222)$. Moreover, multivariate Cox regression model revealed that the ALBI score showed no predictive ability in the BCLC B/ C subgroup $(P=0.122)$.

Prognoses of HCC patients are largely dependent on the tumor characteristics at the time when they undergo surgery. We found differentiation grade and MVI were significant predictors in the Cox regression model.
Compared to patients with early stage $\mathrm{HCC}$, patients with BCLC B/C HCC generally have heavier tumor burdens, as indicated by large tumor sizes, poor differentiation, and vascular invasion [32]. Vascular invasion is an independent risk factor of recurrence and OS, directly correlated with the size of the main nodule and histological differentiation [33, 34]. Differentiation grade indicates a greater likelihood of malignant behavior, resulting in a higher risk of recurrence and metastasis, significantly affect OS. Interestingly, AFP level showed no significance in predicting OS in our study, despite this marker has been reported to predict reaction to locoregional treatments and 
Table 3 Multivariate analyses of factors affecting overall survival across BCLC stage

\begin{tabular}{|c|c|c|c|c|c|c|}
\hline \multirow[t]{3}{*}{ Variable } & \multicolumn{6}{|c|}{ Multivariable Cox regression } \\
\hline & \multicolumn{2}{|l|}{ Total cohort } & \multicolumn{2}{|l|}{$\mathrm{BCLC} 0 / \mathrm{A}$} & \multicolumn{2}{|l|}{$\mathrm{BCLC} B / \mathrm{C}$} \\
\hline & $\mathrm{HR}(95 \% \mathrm{Cl})$ & $P$ & $\mathrm{HR}(95 \% \mathrm{Cl})$ & $P$ & HR $(95 \% \mathrm{Cl})$ & $P$ \\
\hline Age, years & $1.03(0.72-1.48)$ & 0.868 & $1.08(0.61-1.93)$ & 0.791 & $0.79(0.49-1.29)$ & 0.343 \\
\hline Male gender & $0.86(0.53-1.40)$ & 0.541 & $1.10(0.51-2.37)$ & 0.803 & $0.85(0.44-1.65)$ & 0.626 \\
\hline Platelet count, $\times 10^{9} / \mid$ & $1.62(1.10-2.38)$ & 0.014 & $1.90(1.02-3.53)$ & 0.043 & $1.26(0.75-2.11)$ & 0.383 \\
\hline Prothrombin time, sec & $1.00(0.89-1.12)$ & 0.938 & $0.92(0.76-1.11)$ & 0.362 & $1.07(0.92-1.25)$ & 0.379 \\
\hline Serum AFP, ng/ml & $1.09(0.80-1.49)$ & 0.594 & $1.22(0.72-2.05)$ & 0.457 & $0.92(0.61-1.39)$ & 0.677 \\
\hline Tumor size, cm & $1.71(1.21-2.44)$ & 0.003 & $1.95(1.15-3.32)$ & 0.014 & $1.58(0.95-2.64)$ & 0.079 \\
\hline ALBI score & $1.62(1.18-2.22)$ & 0.003 & $2.29(1.39-3.78)$ & 0.001 & $1.42(0.91-2.20)$ & 0.122 \\
\hline Major hepatectomy & $1.33(0.93-1.88)$ & 0.115 & $1.02(0.53-1.95)$ & 0.965 & $1.33(0.86-2.08)$ & 0.201 \\
\hline $\mathrm{MVI}$ & $2.10(1.49-2.97)$ & $<0.001$ & $1.91(1.13-3.01)$ & 0.028 & $1.53(0.89-2.83)$ & 0.046 \\
\hline Differentiation grade & $1.69(1.24-2.30)$ & 0.001 & $1.77(1.08-2.89)$ & 0.024 & $2.03(1.28-3.23)$ & 0.003 \\
\hline MELD score & $0.98(0.90-1.08)$ & 0.727 & $0.98(0.84-1.15)$ & 0.803 & $1.00(0.88-1.14)$ & 0.989 \\
\hline
\end{tabular}

HR hazard ratio, CI confidence interval, BCLC Barcelona Clinic Liver Cancer, AFP a-fetoprotein, ALBI albumin-bilirubin, MVI microvascular invasion, MELD model for end-stage liver disease

outcome of untreated advanced HCC [35, 36]. To summarize, tumor burden is likely to play a more significant role in determining survival time than the underlying liver function in this group of patients. Thus, the ALBI grade alone may not be sufficient to predict OS in BCLC $\mathrm{B} / \mathrm{C}$ stage $\mathrm{HCC}$ patients who undergo liver resection. A possible solution may be integrating the ALBI grade and tumor characteristics to generate a more accurate model to predict OS for this population, which should be performed in the future.

Several limitations exist in the present research. First, most of the patients had an infection of hepatitis $B$ virus as the cause of $\mathrm{HCC}$ in the current research. Our results may not be applicable to Western countries, where hepatitis $\mathrm{C}$ virus infection and alcoholic steatohepatitis are the predominant etiologies of HCC. Second, the reliability of the present research was weakened by its retrospective specialty, single-center data, and comparably small sample capacity. Third, limited by the relatively small sample size, we did not include some variables which might have an effect on PHLF in order to increase the stability of statistical models and the credibility of results. A prospective study design with a large sample and effective controls is needed to expand our findings.

\section{Conclusions}

In summary, our study has verified that the ALBI grade is a significant prognostic factor for PHLF in HCC patients across different BCLC stages. However, for the first time, we found the ALBI grade was only a significant predictor of $\mathrm{OS}$ in $\mathrm{BCLC}$ stage $0 / \mathrm{A}$ patients and was not a good predictor in BCLC stage $\mathrm{B} / \mathrm{C}$ patients.

\section{Additional file}

Additional file 1: Figure S1. Kaplan-Meier curves demonstrating overall survival according to MELD score $<9$ and MELD score $\geq 9$ in the total cohort. BCLC, Barcelona Clinic Liver Cancer; MELD, model for endstage disease. (TIF $801 \mathrm{~kb}$ )

\section{Abbreviations}

AFP: a-fetoprotein; ALBI: Albumin-bilirubin; BCLC: Barcelona Clinical Liver Cancer; CP: Child-Pugh; CSPH: Clinically significant portal pressure; HCC: Hepatocellular carcinoma; INR: International normalized ratio; MELD: Model of end-stage liver disease; OS: Overall survival; PHLF: Post-hepatectomy liver failure

Availability of data and materials

The data are available from the authors if needed.

\section{Authors' contributions}

The manuscript was drafted by ZZQ and revised by $\mathrm{XL}, \mathrm{ZH}$, and ZZQ. ZH and WY designed the clinical study. ZZQ and ZH conducted the data analysis and produced the figures and tables. ZJJ, LQL, and MXY conceived the conception and assisted its design. All authors read and approved the final manuscript.

Ethics approval and consent to participate

Written permission was given up in the current research. This research was authorized by the ethics committee of the Central South University.

Consent for publication

Not applicable.

Competing interests

The authors declare that they have no competing interests.

\section{Publisher's Note}

Springer Nature remains neutral with regard to jurisdictional claims in published maps and institutional affiliations. 
Received: 31 July 2018 Accepted: 25 September 2018

Published online: 16 October 2018

\section{References}

1. Torre LA, Bray F, Siegel RL, Ferlay J, Lortet-Tieulent J, Jemal A. Global cancer statistics, 2012. CA Cancer J Clin. 2015;65:87-108

2. de Lope CR, Tremosini S, Forner A, Reig M, Bruix J. Management of HCC. J Hepatol. 2012:56(Suppl 1):S75-87.

3. Yin L, Li H, Li AJ, et al. Partial hepatectomy vs. transcatheter arterial chemoembolization for resectable multiple hepatocellular carcinoma beyond Milan criteria: a RCT. J Hepatol. 2014;61:82-8.

4. Kokudo T, Hasegawa K, Matsuyama Y, et al. Survival benefit of liver resection for hepatocellular carcinoma associated with portal vein invasion. J Hepatol. 2016;65:938-43.

5. Kokudo T, Hasegawa K, Matsuyama Y, et al. Liver resection for hepatocellular carcinoma associated with hepatic vein invasion: a Japanese nationwide survey. Hepatology. 2017;66:510-7.

6. Ho MC, Hasegawa K, Chen XP, et al. Surgery for intermediate and advanced hepatocellular carcinoma: a consensus report from the 5th Asia-Pacific primary liver cancer expert meeting (APPLE 2014). Liver Cancer. 2016:5:245-56.

7. Farges O, Malassagne B, Flejou JF, Balzan S, Sauvanet A, Belghiti J. Risk of major liver resection in patients with underlying chronic liver disease: a reappraisal. Ann Surg. 1999;229:210-5.

8. Belghiti J, Hiramatsu K, Benoist S, Massault P, Sauvanet A, Farges O. Seven hundred forty-seven hepatectomies in the 1990s: an update to evaluate the actual risk of liver resection. J Am Coll Surg. 2000;191:38-46.

9. Yamanaka N, Okamoto E, Kuwata K, Tanaka N. A multiple regression equation for prediction of posthepatectomy liver failure. Ann Surg. 1984; 200:658-63.

10. Johnson PJ, Berhane S, Kagebayashi C, et al. Assessment of liver function in patients with hepatocellular carcinoma: a new evidence-based approachthe ALBI grade. J Clin Oncol. 2015;33:550-8.

11. Hiraoka A, Michitaka K, Kumada T, et al. Validation and potential of albuminbilirubin grade and prognostication in a nationwide survey of 46,681 hepatocellular carcinoma patients in Japan: the need for a more detailed evaluation of hepatic function. Liver Cancer. 2017:6:325-36.

12. Li MX, Zhao H, Bi XY, et al. Prognostic value of the albumin-bilirubin grade in patients with hepatocellular carcinoma: validation in a Chinese cohort. Hepatol Res. 2017:47:731-41.

13. Wang $Y Y$, Zhong JH, Su ZY, et al. Albumin-bilirubin versus child-Pugh score as a predictor of outcome after liver resection for hepatocellular carcinoma. Br J Surg. 2016;103:725-34.

14. Zou H, Wen Y, Yuan K, Miao XY, Xiong L, Liu KJ. Combining albuminbilirubin score with future liver remnant predicts post-hepatectomy liver failure in HBV-associated HCC patients. Liver Int. 2018;38:494-502.

15. Liu PH, Hsu CY, Hsia CY, et al. Prognosis of hepatocellular carcinoma: assessment of eleven staging systems. J Hepatol. 2016;64:601-8.

16. Pinato DJ, Sharma R, Allara E, et al. The ALBI grade provides objective hepatic reserve estimation across each BCLC stage of hepatocellular carcinoma. J Hepatol. 2017;66:338-46.

17. Toyoda H, Lai PB, O'Beirne J, et al. Long-term impact of liver function on curative therapy for hepatocellular carcinoma: application of the ALBI grade. Br J Cancer. 2016;114:744-50

18. Chong CC, Chan AW, Wong J, et al. Albumin-bilirubin grade predicts the outcomes of liver resection versus radiofrequency ablation for very early/ early stage of hepatocellular carcinoma. Surgeon. 2018;16:163-70.

19. Dong ZR, Zou J, Sun D, et al. Preoperative albumin-bilirubin score for postoperative solitary hepatocellular carcinoma within the Milan criteria and child-Pugh a cirrhosis. J Cancer. 2017:8:3862-7.

20. Bruix J, Sherman M. Management of hepatocellular carcinoma: an update. Hepatology. 2011;53:1020-2.

21. Rahbari NN, Garden OJ, Padbury R, et al. Posthepatectomy liver failure: a definition and grading by the International Study Group of Liver Surgery (ISGLS). Surgery. 2011;149:713-24.

22. Balzan S, Belghiti J, Farges O, et al. The "50-50 criteria" on postoperative day 5: an accurate predictor of liver failure and death after hepatectomy. Ann Surg. 2005;242:824-8 discussion 28-9.

23. Forner A, Llovet JM, Bruix J. Hepatocellular carcinoma. Lancet. 2012;379: $1245-55$.
24. Chen X, Zhai J, Cai X, et al. Severity of portal hypertension and prediction of postoperative liver failure after liver resection in patients with Child-Pugh grade a cirrhosis. Br J Surg. 2012;99:1701-10.

25. Pugh RN, Murray-Lyon IM, Dawson JL, Pietroni MC, Williams R. Transection of the oesophagus for bleeding oesophageal varices. Br J Surg. 1973;60: 646-9.

26. Butt AA, Ren Y, Lo Re V 3rd, Taddei TH, Kaplan DE. Comparing child-Pugh, MELD, and FIB-4 to predict clinical outcomes in hepatitis $C$ virus-infected persons: results from ERCHIVES. Clin Infect Dis. 2017;65:64-72.

27. Schroeder RA, Marroquin CE, Bute BP, Khuri S, Henderson WG, Kuo PC. Predictive indices of morbidity and mortality after liver resection. Ann Surg. 2006;243:373-9.

28. Teh SH, Sheppard BC, Schwartz J, Orloff SL. Model for end-stage liver disease score fails to predict perioperative outcome after hepatic resection for hepatocellular carcinoma in patients without cirrhosis. Am J Surg. 2008; 195:697-701.

29. Stockmann M, Lock JF, Riecke B, et al. Prediction of postoperative outcome after hepatectomy with a new bedside test for maximal liver function capacity. Ann Surg. 2009;250:119-25.

30. Lin XJ, Yang J, Chen XB, Zhang M, Xu MQ. The critical value of remnant liver volume-to-body weight ratio to estimate posthepatectomy liver failure in cirrhotic patients. J Surg Res. 2014;188:489-95.

31. Augustin S, Millan L, Gonzalez A, et al. Detection of early portal hypertension with routine data and liver stiffness in patients with asymptomatic liver disease: a prospective study. J Hepatol. 2014;60:561-9.

32. Sasaki K, Morioka D, Conci S, et al. The tumor burden score: a new "metroticket" prognostic tool for colorectal liver metastases based on tumor size and number of tumors. Ann Surg. 2018;267:132-41.

33. Roayaie S, Blume IN, Thung SN, et al. A system of classifying microvascular invasion to predict outcome after resection in patients with hepatocellular carcinoma. Gastroenterology. 2009;137:850-5.

34. Sumie S, Kuromatsu R, Okuda K, et al. Microvascular invasion in patients with hepatocellular carcinoma and its predictable clinicopathological factors. Ann Surg Oncol. 2008;15:1375-82.

35. Llovet JMPC, Shan M, Lathia C, Bruix J. Biomarkers predicting outcome of patients with advanced hepatocellular carcinoma (HCC) randomized in the phase III SHARP trial. Presidential plenary session, AASLD 59th annual meeting, San Francisco. Hepatology. 2008;48:372A.

36. Llovet JM, Bruix J. Systematic review of randomized trials for unresectable hepatocellular carcinoma: chemoembolization improves survival. Hepatology. 2003;37:429-42.

\section{Ready to submit your research? Choose BMC and benefit from:}

- fast, convenient online submission

- thorough peer review by experienced researchers in your field

- rapid publication on acceptance

- support for research data, including large and complex data types

- gold Open Access which fosters wider collaboration and increased citations

- maximum visibility for your research: over $100 \mathrm{M}$ website views per year

At $\mathrm{BMC}$, research is always in progress.

Learn more biomedcentral.com/submission 